\title{
BUILDING TANGIBLE AUGMENTED REALITY MODELS FOR USE IN PRODUCT DEVELOPMENT
}

\author{
Jain, Karan; Choi, Young Mi \\ Georgia Institute of Technology
}

\begin{abstract}
This paper discusses the issues involved with creating a Tangible Augmented Reality representation of a product so that it can be effectively and validly used to support the product design process. The development of a TAR representation entails building the physical model of the product, a digital model of the product, and developing interactions between the digital and physical representations. Different products have different user interactions, which makes developing TAR representation for products challenging since a new interaction needs to be mimicked. Challenges of developing TAR representations are discussed by investigating TAR representations of four consumer products: a space heater, a MP3 player, an electric can opener, and a seat assist cushion. The goal of TAR representations is to not only communicate the functionality of a product but also to improve the time efficiency of prototyping of physical products. Hence, having a good understanding of the user interactions that need to be developed and planning the representation for construction in the modeling software and AR software is crucial to making the process of developing a TAR representation efficient.
\end{abstract}

Keywords: Case study, Simulation, Product modelling / models, Tangible Augmented Reality

\section{Contact:}

Jain, Karan

Georgia Institute of Technology

School of Industrial Design

United States of America

kjain33@gatech.edu 


\section{INTRODUCTION}

The goal of this paper is to enumerate some of the challenges that may be encountered when attempting to construct a Tangible Augmented Reality (TAR) representation of a product suitable for use in usability testing or for gathering end user feedback. While utilizing TAR presents many potential advantages, the technology has only recently become more widely available and ways in which it might be effectively and validly utilized within the product design process has not been broadly studied. The product development process relies on eliciting feedback from users to ensure that the product is meeting user needs and wants. During the early stages of the design process, designers often do not have enough information about user needs (Bruseberg and McDonagh-Philip, 2002). Design representations such as storyboards, sketches, and digital renderings are used to communicate product concepts since creating highly detailed models of early concepts is not feasible (Ray and Choi, 2016). However, users may not be able to accurately visualize a product from an abstract concept (Leonard Barton, 1992), leading to more inaccurate opinions of users during early stage user feedback sessions. Further, these design representations are intangible and do not allow for communicating product functionality, accurately. Detailed input from users can be elicited by testing detailed physical prototypes, particularly for subjective attributes such as aesthetics and emotional appeal, ergonomics and usability, product integrity or craftsmanship (Srinivasan, Lovejoy et al., 1997). Appearance models is an example of a high fidelity design representation that is used to evaluate the intended form of a design concept (Evans, 2002). However, a drawback of these prototypes is that they cannot be built during the initial stages of the design process since product specifications and criteria are not well defined at this stage. Such representations can only be made by designers, later in the process, once concept details are outlined. Updating representations to account for design defects, later in the design process, is costlier and time consuming (McGrath, Anthony et al., 1992). TAR is an augmented reality (AR) related technology where a digital model is superimposed on a physical representation of a product. Users physically interact with the physical model as they would with the real thing but all views of the product such as interface and results of interactions/actions are rendered through the virtual interface. Such a design representation provides an opportunity to communicate abstract concepts along with tangible interactions to better gather user feedback, early in the design process. This paper discusses the process of developing design representations in Tangible Augmented Reality and highlights some challenges faced in the development of TAR representations for four different products.

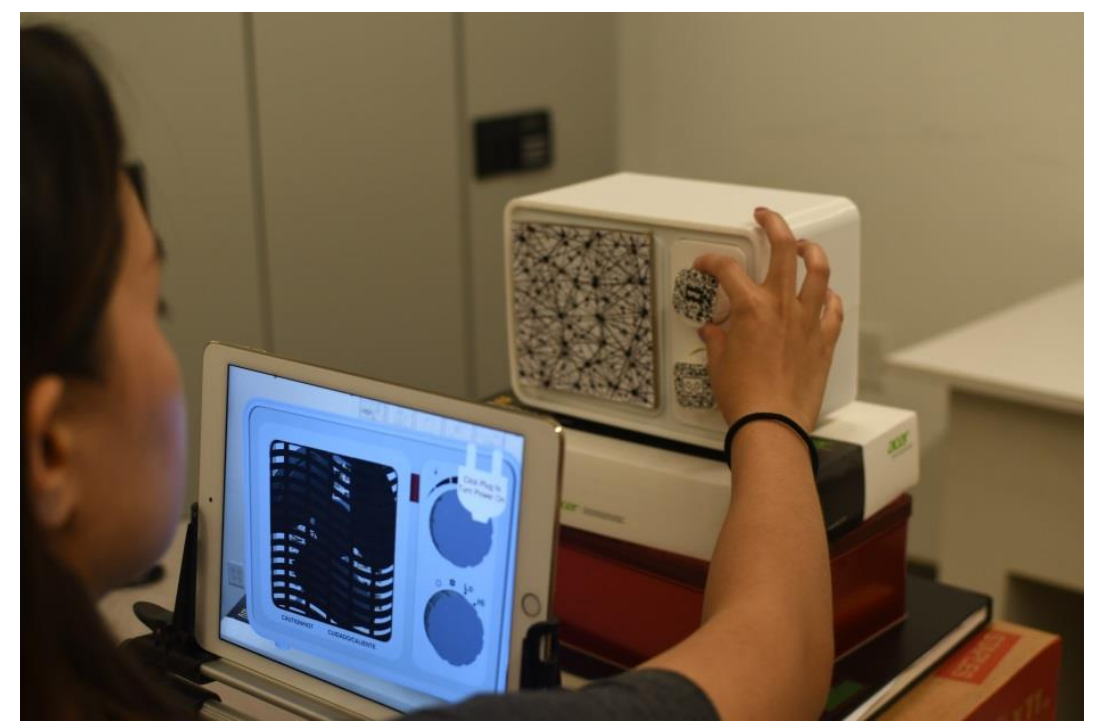

Figure 1. TAR representation of a space heater.

Usability testing is one of the most widely used and important methods for evaluating product design (Lewis, 2006). Usability is the effectiveness, efficiency and satisfaction by which users can achieve specific goals in an environment (ISO, 1998). TAR representations can be extremely valuable in usability evaluation because it can not only communicate details of appearance, scale, and weight but also communicate natural interactions with the product and in turn convey the features and functionalities of the product, accurately. Every product has a different interaction and a different 
response to the user interaction. Therefore, developing a TAR representation for every product presents a new challenge while developing the natural interaction with the physical representation. Further discussion about challenges of building a TAR representations and some implementation solutions are discussed through the examples of TAR representations of a space heater, a MP3 player, an electric can opener, and a seat assist cushion.

\section{CONSTRUCTION OF TAR REPRESENTATION}

The construction of a TAR representation is generally made up of three parts: building a physical representation, 3D modelling all visual components of a product, and building interactions between the physical and digital components. The physical model of a TAR representation should not need to be built to a high level of fidelity since the texture and material appearance of the product is communicated through visuals from the virtual medium. The physical representation should mainly communicate scale, weight, and physical interactions. The mechanisms on the physical representation need to closely resemble the tangible feedback given on the real product. The digital representation should communicate the appearance of a product as accurately as possible.

The goal of the examples presented in this paper was to build a TAR representation that is as close as possible with respect to the physical and visual aspects of an existing product. The 3D models of the examples presented in this paper were developed in Rhinoceros 5. Interaction between the digital model and the physical model were developed in Unity 3D with the Vuforia SDK and an iPad was used to view the superimposition of the digital model on the physical representation. Image targets on the physical representation were tracked by the AR software to render product visuals at defined locations, on the virtual medium, relative to the physical representation. An example of this setup is shown in Figure 1. A physical model (plain white in this case) the same size and weight of a space heater product is shown. The only markings are unique 'targets' which the AR software uses to know the spatial location and position of particular components of the model. There are three in this case, two knobs and a fan. The user is able to interact with the physical model, but all of the detail (color, finish, words, markings, etc) are shown through augmented view rendered via the tablet.

Rhinoceros 5 was chosen for product modelling because of its easy to use interface, ease of learning for polygon modelling (Dönmez, 2014), and availability of the software for both macOS and Windows. Modelling for Unity 3D can also be done in Autodesk 3Ds Max and Fusion360 because of their capabilities of exporting a model as a .fbx or .obj format to work with Unity 3D. Fiducial markers were used for tracking of objects in the field of view but future work should look into development in feature based tracking to make the TAR system more robust. The Vuforia SDK is an excellent tool for fiducial marker tracking because it supports both 2D and 3D targets and also allows for multi target configurations (Amin and Govilkar, 2015). Further, it provides efficient tracking in low light conditions and has the capability of extended tracking. It also has a easy to use Target Management System that allows for uploading images that should be used for marker tracking. Vuforia was initially chosen as the AR SDK for development because it supports Android, iOS, and Unity3D Editor. There are many other AR SDKs available, like ARCore, ARKit, and ARToolKit (Glover, 2018). The drawback with ARCore is that it only supports Android. ARKit cannot be compiled on Windows for Mac and requires a macOS to even test the code. Hence, initially it was decided to not use this SDK. ARToolKit is open source software that has a large variety of function, which makes it a little challenging to integrate the library. This SDK was not used because it would take more time to learn the SDK as compared to Vuforia. Unity was chosen as the game engine for development because the Vuforia SDK is well integrated with Unity 3D. Further, the team had prior knowledge of scripting in C\#, which made Unity a more preferred option over Unreal Engine. Unity also has a large online support community because the software has been free for personal use for a lot longer as compared to Unreal Engine (Dickson et al., 2017).

\section{ChALLENGES IN THE CONSTRUCTION OF TAR REPRESENTATIONS}

The biggest challenge of developing a TAR representation is developing interactions that mimic the natural interaction with the real product as accurately as possible. Hence, the development of a TAR representation should be planned around the user interactions that need to be developed for the representation. A few important issues to consider in the development of a TAR representation are discussed below: 
1. Barriers to natural interaction. A limitation in mimicking the natural interaction accurately is the use of image targets for rendering product visuals. If an image target is covered or moved away from the view of the camera, the virtual medium will not render the product visuals and mapping between physical and digital elements will be lost. Due to this, users have to avoid covering the image targets while interacting with the physical representation, which may cause inconsistencies and unintended behaviour in the way that natural interactions work. Further investigation needs to take place on how to develop TAR representations without the use of image targets in order to represent natural interactions more accurately.

2. Effect of object hierarchy, in Unity 3D, on object position and alignment. If an image target is used to render product visuals on the virtual medium then objects in Unity 3D have to be placed as children of the parent image target. Placing an object as a child of a parent object will set its position relative to the parent object and not relative to the world space. If there are multiple objects in a scene, placed under their respective parent objects, then their alignment and positioning, relative to one another, is difficult to maintain accurately since the objects are not moving relative to a common origin. An effort should be made to place objects under one parent object in order to maintain the desired position of objects relative to one another.

3. Setting correct parameters in 3D modelling software. Parameters such as coordinate position, pivot point, materials, etc. of the 3D model that are set in the modelling software are imported into Unity $3 \mathrm{D}$ and any scripts written for the model will use this metadata. For example, if the object has a specific position relative to the origin in the modelling software, it will have the same position relative to the origin of the world space or parent object (if placed as a child of the parent) when it is imported into Unity 3D. Being cognizant of the parameters set in the 3D modelling software will make debugging of scripts easier while developing interactions and motion for objects in Unity 3D. Secondly, parts of the 3D model can be grouped together, as one solid body, if they are static and should remain as separate solid bodies if they are dynamic. Grouping parts in the modelling software will help maintain an organized object hierarchy in Unity 3D.

4. Consideration of digital and physical objects. The purpose of a TAR representation is to communicate the functionality of a product and the interactions with the product. To communicate functionality clearly, it is important to consider how the use of the product affects other elements in the product's environment or what other object the product depends on in order to be used in a particular context. For example, in the usability test of a television remote, it would be important to test the feedback on a television set when the power button is pressed on the remote. Such tests make it important to consider how other products should be represented in a TAR representation. Is it required to develop physical and digital representations of these other products or can the real products be used to communicate the feedback accurately? Having more digital objects will entail developing more interactions, which increases the time of prototyping and reduces the efficiency of using a TAR representation.

\section{CASE STUDIES OF TAR REPRESENTATIONS}

\subsection{Space heater}

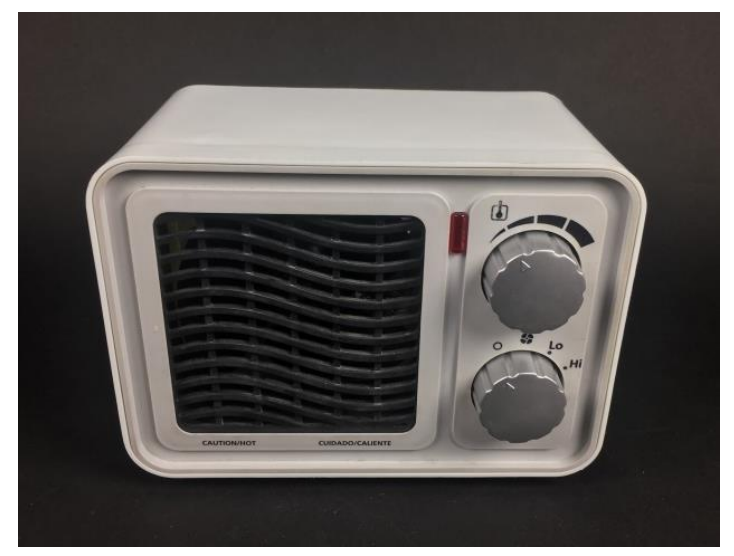

Figure 2. Sunbeam model SFH5264MW heater. 
Figure 2 shows the Sunbeam model SFH5264MW space heater whose TAR representation was developed for the study. The top knob on the heater controls the temperature and the bottom knob controls the mode, which are off, fan only, low heat, and high heat. The heater has several labels on it which provide instructions related to the functionality of the heater. The challenge in the development of the TAR representation for this product was learning how to develop a representation using multiple image targets.

Figure 3 shows the physical and the digital component of the TAR representation of the space heater. The physical representation was put together by a combination of fabrication techniques, where the front and back surfaces, which are laser cut acrylic, are held together by a 3D printed bounding shell. The knobs on the physical model are also 3D printed. The mechanism on the bottom knob of the heater was accurately modelled and 3D printed to give the haptic feedback of clicking in three positions, as it is represented in the real product.

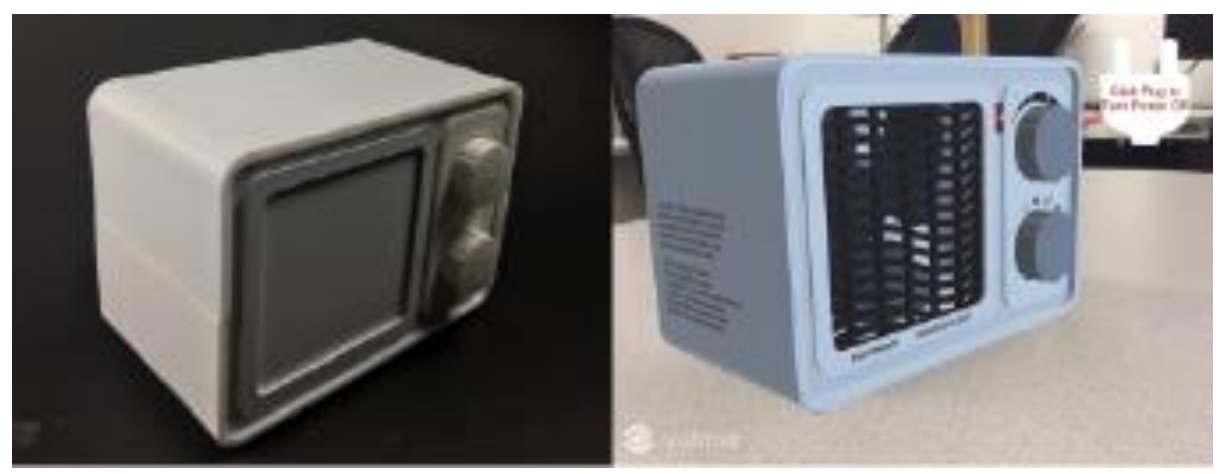

Figure 3. Physical and digital representations of the space heater TAR.

The 3D model of the heater has multiple parts: the bounding body, the grill, the fan, and the knobs. All static parts were combined into one solid body before importing the model into Unity 3D. The dynamic parts, the fan and the knobs, were separate parts in the digital model. The physical representation of the space heater has three image targets: one target for the body and the fan and one target for each of the knobs. The digital model of the knobs could be imported as separate objects and placed under their respective parent image targets in the hierarchy in Unity 3D, however, this caused an issue with maintaining consistent alignment of the objects in Unity 3D. The main heater body is placed relative to its own image target and each knob is placed relative its respective target. Even if the position of the objects is set, in Unity 3D, to align with one another, a change in position of any of the targets in the real world will cause a change in the position of its specific child object because the positions are not relative to the world space. This causes a problem in maintaining the position of knobs relative to the heater body because future updates may need to be made on the physical model to better represent the real product. To fix this issue, the knobs of the heater 3D model were not imported as separate objects. Their position was set accurately, relative to the heater body, in the modelling software and they were just made a different solid body. All static (heater body and grill) and dynamic parts (knobs and fan) were imported into Unity 3D as one object and was made the child of one parent image target. This ensured alignment consistency of the product visuals since the coordinate position of all objects was set relative to the coordinates of one parent. The two smaller image targets on the physical knobs were still used to develop the rotation of the digital knobs. The Euler angle of each of the image target on the physical knobs was mapped to the Euler angle of the respective digital knobs, thereby, changing the rotational position of the digital knob when the physical knob was rotated. This ensured that the coordinate position of the digital knobs did not change (because they were on the same image target as the body) and their rotation mapped with the rotation of the physical knobs without any lag. Since the Euler angle values are applied to the metadata of the digital knobs, it had to be ensured that the pivot points of each of the knobs were set to the centre of their respective knobs in the modelling software. If the pivot point was off centred, then the digital knob would still map accurately to the rotation of the physical knob, but it would rotate around an axis that is off centred.

The rotation motion of the fan was animated in Unity 3D. To represent the motion of the fan more accurately, the fan was animated to speed up, maintain a constant rotation, slow down, and finally stop once the heater was turned off. Sounds for the fan rotation and knob click were also implemented in 
Unity 3D to better mimic the feedback given in the real product. Another aspect of the real space heater was the power cord, which had to be plugged into a power source to turn on the heater. When the heater was turned on, a light on the front surface of the heater came on. This interaction was mimicked in the TAR representation with a virtual button. Users tapped on a button, shaped like a power plug, to turn the digital heater on or off, which also toggled the appearance of a light on the front surface of the heater. To develop the light visual, a point light source was placed behind the bulb surface on the body of the digital space heater. This point source was placed behind the surface in order to give a more translucent effect and represent the colour of the light in the real product more accurately.

One of the biggest challenges of developing a TAR model is accurately representing the natural interaction with the product. Since image target based AR is being used in the development of the TAR representations, the image target must always be in view of the camera in order to render the product visuals. Therefore, while interacting with the physical knobs, users had to ensure that they do not cover the image target. This caused inconsistencies and unintended behaviour in the way that interactions work. Further, if the image target was covered, mapping between the physical knobs and digital knobs was lost and the rotation of the digital knobs did not update. Another challenge with the image targets was that the AR software does not read small image targets consistently. So, mapping between the digital and physical knobs was lost at certain angles because smaller image targets were placed on the physical knobs. The AR software cannot render the back surface of the heater if the physical representation is turned around to an angle where the software loses tracking of the target. Even though lighting in Unity 3D was set to communicate an accurate appearance of the digital model, the lighting in the software can never accurately replicate the lighting in the physical environment. Hence, a difference in the appearance between the digital model and the real product will always be noticed when placed next to one another.

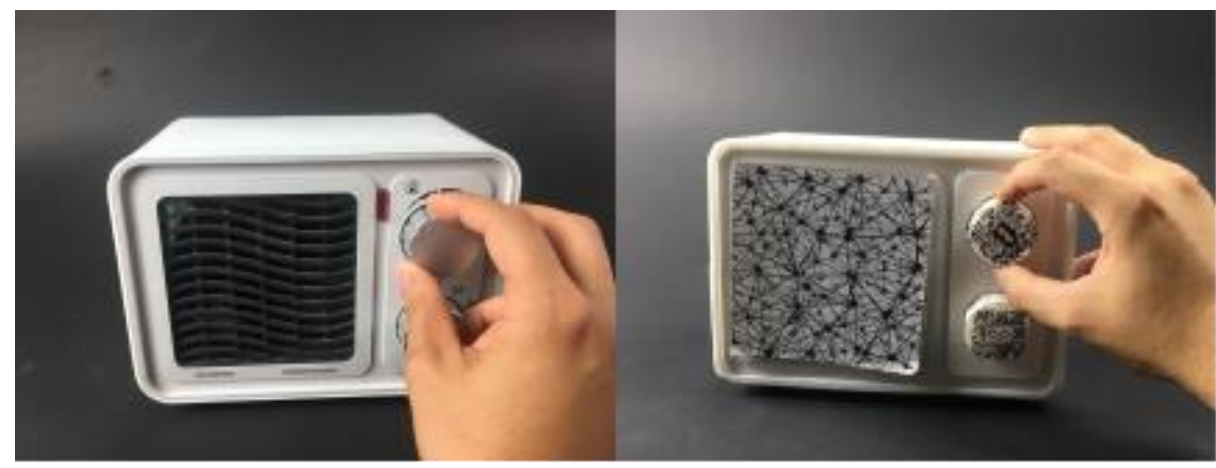

Figure 4. Interaction with the TAR representation vs interaction with the real space heater.

\subsection{MP3 player}

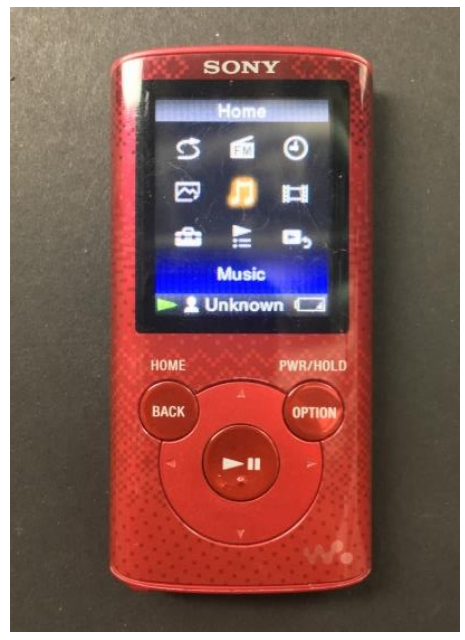

Figure 5. Sony Walkman NWZ-E463.

Figure 5 shows the Sony Walkman NWZ-E463 whose TAR representation is under construction for the study. The product consists of a digital interface and interactions with physical buttons on the body of the device. This MP3 player can store and play audio tracks, has a FM feature, and can store images. These features need to be developed in the TAR representation of the MP3 player in order to 
communicate the functionality of the product. Using image targets to develop natural interactions with the physical representation poses the greatest challenge in the development of the TAR representation of the MP3 player.

The discussion about the digital model is based on the AR representation of the MP3 player. In the AR representation, users interact with the digital model on the 2D interface of the iPad. The development of a TAR representation for this product poses a completely different challenge because a user interface (UI) needs to be developed in the digital representation and the interaction is a press down interaction instead of a rotation interaction. The UI was replicated by taking pictures, under the same lighting condition, of the different screens on the actual device. These images were imported into Unity $3 \mathrm{D}$ and were switched out based on the input command. This made the process of replicating the UI faster and communicated the user flow to a level that was easily understood by participants.

Another feature that was developed in the digital model was playing music and FM. Sound files were imported into Unity 3D and a file was played when users reached the respective UI screen and tapped the "play" button on the digital model on the 2D interface. A real FM feature was not built into the model but a sound track played to mimic the working of the FM feature.

As discussed earlier, the use of image targets to render and update product visuals is one of the biggest challenges in the development of a TAR representation. The physical interaction with the TAR representation is a press down interaction; however, if an image target is placed on the physical button, it will be hidden by the user's finger and will not be able to update any parameters in the digital model. This scenario entails a great challenge since a lot of physical interactions with products involve a press down interaction. Further investigation needs to be done into detecting interactions using more advanced methods of computer vision in order to develop TAR representations for products that entail interactions where the image target may be covered.

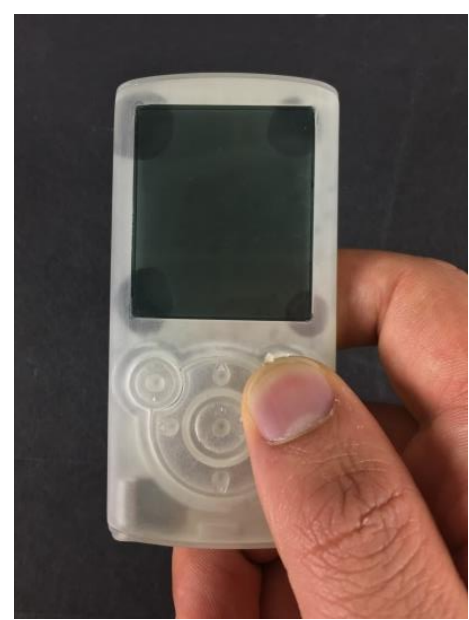

Figure 6. Natural interaction with MP3 TAR physical model.

\subsection{Can opener}

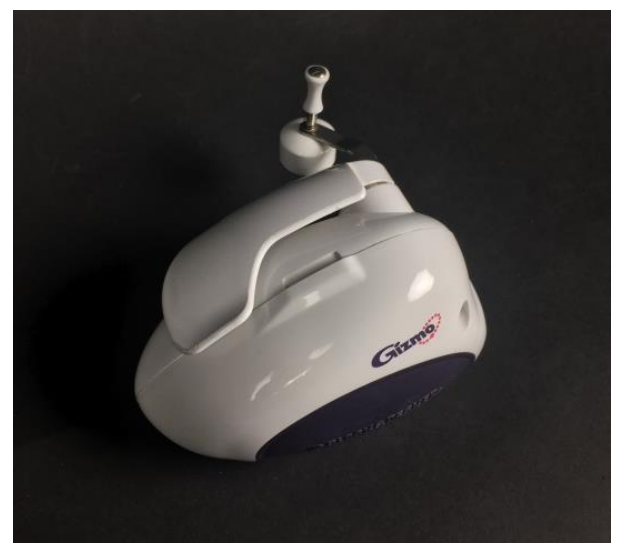

Figure 7. Black and Decker GC200 Gizmo. 
Figure 7 shows an image of the Black \& Decker Gizmo Can Opener whose TAR representation is under development. The cordless opener can be clamped onto cans of different sizes and automatically moves around the can separating the lid from the can. The opener is held onto the can with a swing out magnet. The challenge in the development of the TAR representation of the opener is considering how to represent a can in order to communicate the functionality of the can opener more accurately. The TAR representation of this product will not only entail a physical and digital representation of the opener but also a physical real can which has a digital model of a can superimposed on it through the virtual medium. Users will interact with the physical model of the opener, which will be clamped to the can, and the digital opener will move around the digital can and show how the lid is separated. The opener is hands free and does not require the user to interact with the opener while it is moving around the can. Hence, no tangible feedback other than the resistance of pressing the button, which activates the can opener, is required in the physical model of the TAR representation. Consideration of this supporting product, the can, poses a challenge in the development of this representation.

The opener has an organic shape, which possess some challenge in modelling the product to represent the real product as closely as possible. It is important to get the curves right because when lighting is placed in Unity $3 \mathrm{D}$, the shadows on the product closely communicate its appearance and having any inconsistencies in the form may result in inaccurate rendering of the product due to lighting and shadows. Next, the interaction with the opener is a push down interaction, which like the MP3 TAR representation, will be difficult to detect if an image target is being used. However, the area of the surface with which users interact is large, as compared to the MP3 player, and an image target can be placed at a location on the interaction surface which will not interfere with the user interaction and its motion can be detected to trigger the digital model of the can opener. Since this representation requires a can as well as the opener, a decision needs to be made on whether a real can will be used or a can should be fabricated. Since the focus of the TAR representation is to communicate the functionality of a product and natural interactions with the product, it is not necessary to fabricate the supporting object in this scenario. A real can will be used with an image target placed on its top surface, which will render the digital model of the opener and the can, thereby increasing the efficiency of prototyping. However, using a real can possess an additional challenge of communicating how the lid is being separated from the can when the opener is moving around the can. In order, to achieve this a digital can will have to be modelled and an animation will be developed to show the separation of the lid from the can when the opener is moving around the can. Further, like the real product, a real magnet can be used in the physical model of the TAR representation in order to clamp the physical model of the representation onto a real can. Mimicking the real product in such ways adds more accurate feedback to interactions with the physical model and also helps to represent the weight of the real product more closely.

One of the biggest challenges with this type of representation is that the digital model is superimposed on the physical model, however, the digital model will move and the physical model will stay stationary. Once the digital model moves from its original position, the physical model will still be seen through the virtual medium, thereby causing a discrepancy in the product visuals. A solution needs to be developed on how to hide the physical model when the digital model is not perfectly overlaid on the physical model. Another interesting aspect of this system is that the can vibrates due to the interaction between the blade on the opener and the lid. In this scenario, the user is not holding onto the can and the vibration does not introduce something that is native to the interaction. Adding vibration to the physical model of the TAR representation will entail making something that is not natural to the regular interaction of the product; hence, such feedback is not necessary to develop in a TAR representation. It is important to understand what interactions and feedback communicate natural interactions and functionality in order to make the process of developing TAR representations more efficient. 


\subsection{Seat assist cushion}

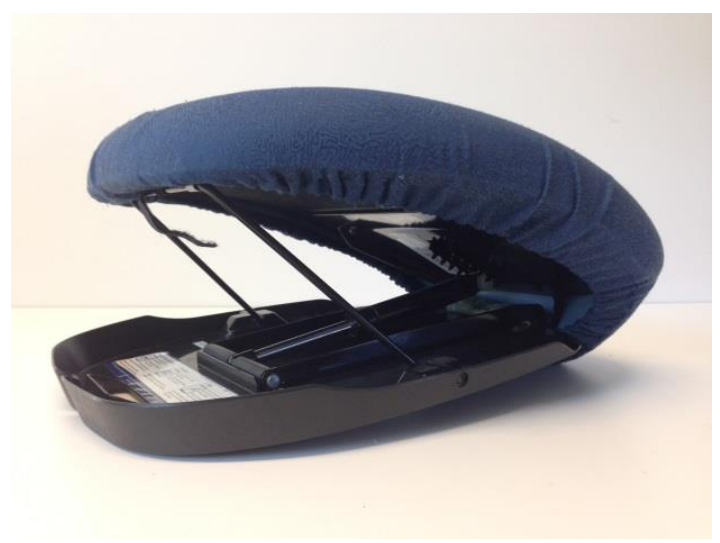

Figure 8. Carex UpEasy Seat Assist.

Figure 8 shows the Carex UpEasy Seat Assist whose TAR representation is currently being planned out for the study. This portable lift assist device can be used on various different kinds of seats for increased mobility and also has a built-in carry handle. It is self-powered with a hydro-pneumatic gas spring, which releases slowly as the user begins to stand. The TAR representation of this product poses an interesting challenge because the user does not look at the product while interacting with it.

The development of the physical representation of the cushion is extremely challenging. Not only is a soft good feel required to be mimicked but also a mechanism will have to be developed for the pneumatic spring. Further, the physical representation will have to be functional, so it will have to be developed robustly in order to support the weight of a user. Such challenges call for further consideration in the development of the physical representations. Since, users do not look at the product while interacting with it, the real cushion itself could be used instead of developing a physical representation from scratch. The real cushion will have to be painted white in order to reduce the fidelity of the physical representation. Since, users never look at the motion of the pneumatic spring, an animation for the spring does not need to be developed, however, an animation for the top surface of the cushion moving up or down will need to be developed since this part of the cushion is in the users view. Further work is being done to better understand the use scenarios for the seat assist and how users actually interact with this product in order to develop the TAR representation for this product.

\section{CONCLUSION}

This paper outlined some challenges in developing a TAR representation for product evaluation. The construction of a TAR representation requires building a physical representation, a digital representation, and developing interactions between the physical and digital representations. Challenges and considerations from the development of TAR representations of certain products were discussed in order to highlight how TAR representations of products vary because of differences in user interaction. The hierarchy of objects in Unity 3D should be considered and planned in order to ensure proper alignment of objects. Further, being cognizant of parameters set in the 3D modelling software allows for easier debugging of scripts in Unity 3D. The biggest challenge in representing the natural interaction accurately is the use of image targets. Further, investigation needs to be done on how to build TAR representations without image targets. Considering how to represent any supporting products in the development of a TAR representation is also an important factor to ensure efficient use of time. In order to make the process of development of a TAR representation more efficient, it is important to understand what interactions and feedback communicate natural interactions with the product and functionality of the product accurately.

\section{ACKNOWLEDGEMENTS}

The contents of this paper were developed in part under a grant from the National Institute on Disability, Independent Living, and Rehabilitation Research (NIDILRR grant number 90RE5007-01-00). NIDILRR is a Center within the Administration for Community Living (ACL), Department of Health and Human Services (HHS). The contents of this paper do not necessarily represent the policy of NIDILRR, ACL, HHS, and you should not assume endorsement by the Federal Government. 


\section{REFERENCES}

Amin, D. and Govilkar, S. (2015), “Comparative Study of Augmented Reality Sdk's”, International Journal on Computational Science \& Applications, Vol. 5 No. 1, pp. 11-26. https://dx.doi.org/10.5121/ijcsa.2015.5102.

Bruseberg, A. and McDonagh-Philip, D. (2002), "Focus groups to support the industrial/product designer: A review based on current literature and designers' feedback." Applied Ergonomics Vol. 33 No. 1, pp. 27-38.

Dickson, P. E. et al. (2017), “An Experience-based Comparison of Unity and Unreal for a Stand-alone 3D game development course”, pp. 70-75. https://dx.doi.org/10.1145/3059009.3059013.

Dönmez, S. (2014), "Computer Aided Industrial Design Software Selection in Industrial Product Design Education at Turkey Using Expert Choice Program", Procedia - Social and Behavioral Sciences, Vol. 106, pp. 682-689. https://dx.doi.org/10.1016/j.sbspro.2013.12.078.

Evans, M. (2002), “The integration of rapid prototyping within industrial design practice”, from http://hdl.handle.net/2134/5155

Glover, J. (2018), Unity 2018 Augmented Reality Projects, Packt Publishing Ltd., Birmingham, pp. 13-14

ISO (1998), "ISO 9241-11", Ergonomic Requirements for Office Work with Visual Display Terminals (VDTs)Part Vol. 11, Guidance on Usability.

Leonard-Barton, D. (1992), "Core Capabilities and Core Rigidities: A Paradox in Managing New Product Development", Strategic Management, Vol. 13 No. 1, pp. 111-125.

Lewis, J. R. (2006), "Usability testing”, Handbook of Human Factors and Ergonomics. G. Salvendy: pp. 1275-1316.

McGrath, M. E., Anthony, M. T. et al. (1992), Product development: success through product and cycle-time excellence, Butterworth-Heinemann.

Srinivasan, V., Lovejoy, W. S. and Beach, D. (1997), "Sharing user experiences in the product innovation process: Participatory design needs participatory communication", Creativity and Innovation Management Vol. 16, pp. 35-45.

Samantak, R. and Mi, C. Y. (2017), "Employing design representations for userfeedback in the product design lifecycle", Proceedings of the International Conference on Engineering Design, ICED, 4(DS87-4), pp. 563-572. Available at: https://www.scopus.com/inward/record.uri?eid=2-s2.0$85029741860 \&$ partnerID $=40 \&$ md5 $=5906 \mathrm{c} 0959 \mathrm{bf5c5e} 07 \mathrm{aac} 5679 \mathrm{~cd} 1 \mathrm{ee} 29 \mathrm{f}$. 